\title{
GESTIÓN DEL CONOCIMIENTO EN EMPRESAS Y ORGANIZACIONES SOCIALES PRODUCTIVAS EXITOSAS. UN ESTUDIO EN EL SURESTE DE MÉXICO
}

\author{
Francisco Gerardo Barroso Tanoira*
}

\begin{abstract}
Barroso-Tanoira F.G. Gestión del conocimiento en empresas y organizaciones sociales productivas exitosas. Un estudio en el sureste de México. Hitos de Ciencias Económico Administrativas 2013;19 (55): 103-114.
\end{abstract}

\section{RESUMEN}

Objetivo: Identificar las estrategias de gestión del conocimiento de empresas y Organizaciones Sociales Productivas (OSP) exitosas y evaluar si el desempeño exitoso de éstas corresponde a su gestión del conocimiento.

Material y método: El estudio fue cualitativo y constó de dos fases; en la primera participaron los directivos o responsables de 20 empresas y de 4 OSP de reconocido éxito con sede en Mérida, Yucatán, seleccionadas por expertos. Asimismo, para identificar las estrategias de gestión del conocimiento, se les administró un cuestionario diseñado para tal efecto. Con la información resultante se diseñaron escalas para evaluar dicha gestión.

Resultados: Aunque adquieren conocimiento de manera eficaz, la mayoría de las empresas son consumidoras de conocimiento técnico, pues el que generan se relaciona con la operación del negocio. Se enfocan en la eficiencia de procesos y la transferencia de conocimiento es limitada. El éxito empresarial corresponde a "muy buenos» o "excelentes» calificaciones de gestión del conocimiento en las manufactureras y las de servicio, pero de «regulares» a «excelentes» en las comerciales, siendo estas últimas "deficientes» en cuanto a transferencia del conocimiento. Para las OSP el resultado fue de «regular», y para la caja popular fue «deficiente».

\begin{abstract}
Barroso-Tanoira F.G. Knowledge management in successful productive companies and social organizations. A study in the Southeast of Mexico. Hitos de Ciencias Económico Administrativas 2013;19 (55): 103-114.
\end{abstract}

\section{ABSTRACT}

Objective: Identify the knowledge management strategies in successful and productive enterprises and social organizations, as well as evaluate if such successful performance corresponds to their knowledge management.

Material and method: The study was qualitative and consisted of two stages. In the first one, directors or chairmen of 20 companies and 4 social productive organizations in Merida, rated as successful by experts, participated. In order to identify the strategies of knowledge management, a questionnaire previously designed for this purpose was administered to them. With the resulting information, scales to evaluate such management were designed.

Results: Although they acquire knowledge effectively, most of the enterprises are consumers of technical knowledge because the one they generate is related to the business operation. They focus their attention on the processes efficiency and the transference of knowledge is limited. The successful performance corresponds to «very good» or "excellent» scores in knowledge management procedures in the manufacturing and service firms, but from «regular» to «excellent» in the commercial ones, having these last ones «deficient» scores in knowledge transference. For the social productive organizations the result was "regular», and for the social credit or financial institution, it was «deficient».

*Doctor en Ciencias Administrativas. Universidad Anáhuac Mayab. Mérida, Yucatán. 
Conclusiones. Las empresas exitosas en mercados de mucha competencia y dinamismo, presentan una gestión del conocimiento de «muy buena»a «excelente», orientándose a la innovación, mientras que aquéllas en mercados de mucha competencia pero menos dinámicos presentan un esquema «regular», orientándose más a la operación, pero con limitada innovación. Finalmente, las organizaciones con poca competencia en el mercado, como las OSP, califican de «regular» a «deficiente», centrándose más en el uso y sin hacer muchos esfuerzos por adquirir nuevo conocimiento o transferir el que tienen, operando en un nicho o segmento tipo «zona de confort».

Palabras claves: Conocimiento. Información. Gestión del conocimiento. Adquisición del conocimiento. Uso del conocimiento. Transferencia del conocimiento.
Conclusions. Successful enterprises in highly competitive and dynamic markets presenta «very good» or «excellent» knowledge management oriented to the innovation, while those in highly competitive markets but less dynamic, present a «regular» scheme, more oriented to the operation, but with limited innovation. Finally, organizations with low competitiveness in the market, such as the social productive organizations, were evaluated as «regular» or "deficient», focusing their orientation more in the use of knowledge and making very little effort for acquiring new knowledge or for transferring the ones they have, and in such way, they operate in a «comfort-zone-like» niche or segment.

Key words: Knowledge. Information. Knowledge management. Knowledge acquisition. Knowledge usage. Knowledge transference.

DIRECCIÓN PARA RECIBIR CORRESPONDENCIA: Correo electrónico:francisco.barroso@anahuac.mx, fbarroso_tanoira@yahoo.com.mx

ara las organizaciones actuales, el conocimiento es el principal activo que poseen las personas, instituciones, empresas y naciones para adaptarse, sobrevivir y desarrollarse en el nuevo orden mundial. Está en proceso de reemplazar al capital financiero como principal condicionante del desarrollo de las sociedades, hasta el punto en que diversos autores han descrito su creciente importancia en el nuevo contexto mundial. Drucker (1993) plantea que en la economía el conocimiento, éste no solamente se ha sumado como uno más de los recursos tradicionales de la actividad productiva (tierra, trabajo y capital), sino que se ha convertido en el único recurso que garantiza la sustentabilidad económica en el presente y en el futuro, lo que es compartido por diversos autores como Castells (1997), Garvin (2000) y Argyris (1992). Por esto, las empresas y organizaciones en general han visto en la gestión del conocimiento una oportunidad para satisfacer las necesidades del mercado y ser más competitivas, por lo que desde su visión y misión deben considerar y establecer las pautas para armonizar la creación, recolección, preservación y difusión del conocimiento en un contexto de responsabilidad social y de contribución al desarrollo integral local y nacional, por un lado, y de congruencia con los cambios a nivel global, por el otro.

\section{Tipos de capital en las organizaciones}

En la era de la información, el capital que adquiere mayor importancia en el análisis estratégico es ciertamente aquel que implica activos intangibles como el capital humano (conjunto de competencias que el trabajador pone a disposición de la empresa), por lo que las competencias del personal (conocimiento más habilidades más actitudes), la tecnología de la información y los incentivos a la innovación, pueden desempeñar un papel preponderante en la creación de valor (Bautzer, 2010). Dicho capital no financiero puede clasificarse de la siguiente manera:

\section{Capital intelectual}

Es la competencia, entendida como la suma de «saber» (conocimiento), «saber hacer» (habilidades) y «saber ser» (actitudes). Los activos basados en el conocimiento, tienen que ser evaluados con extrema cautela porque su impacto en el destino de cualquier negocio es enorme; es una cuestión de supervivencia y de competitividad, no de vanidad académica. Es el capital intelectual y no el financiero el que determina el valor del mercado de una organización (Bautzer, 2010).

Los nuevos modelos de negocios apuntan hacia la necesidad de la gestión del capital intelectual como factor determinante para el desarrollo sostenible, y a este tipo de capital se asocian los temas éticos tan intensamente en boga en una sociedad informacional 
que aún no alcanza su plena madurez en relación con sus procesos humanos. El desafío del capital intelectual está en procurar la adecuación entre el hombre y su trabajo, buscando una mayor adaptabilidad del sujeto a la naturaleza y a las exigencias del mercado, así como mayor sociabilidad, desarrollo afectivo y solidez individual. Por ello, todo el personal, sin considerar su posición jerárquica, debe contribuir a la formación del capital intelectual, para lo que debe existir una política clara, definida y, fundamentalmente, un sistema de información que sustente sus acciones, convirtiendo el conocimiento individual almacenado o tácito (Nonaka y Takeuchi, 1995), en conocimiento útil y disponible. Esto es conocimiento socializado compartido y sustento para generar nuevos conocimientos, lo cual crea un diferencial comúnmente llamado «inteligencia competitiva», con posibilidad de transformarse al final en rentabilidad (Bautzer, 2010).

\section{Capital estructural}

Incluye las estrategias, las estructuras y sistemas, las rutinas y los procedimientos que pertenecen a la organización, pudiendo ser reproducidos y compartidos. Se trata pues de las aptitudes organizativas de la empresa para satisfacer los requerimientos del mercado, pues su función es reunir, organizar, refinar y distribuir los conocimientos existentes en forma eficiente. De aquí, Stewart (1998) sugiere tres propósitos fundamentales del capital estructural: (1) potenciar por medio de la especialización, pues de esa manera se logra reducir los esfuerzos de muchos otros; (2) simplificación y automatización, facilitando el procesamiento de datos y la administración de la información, y (3)administración de inventarios (stocks), lo cual consiste en proporcionar conocimientos solamente sobre pedido para minimizar el costo del capital intelectual.

\section{Capital relacional}

Es el valor de las relaciones y contactos de la organización con la gente con la cual ésta hace negocios, pues es precisamente en la relación con los clientes, proveedores y otros involucrados, que el capital intelectual se convierte en dinero, por lo que se define como la relación progresiva con las personas u organizaciones con las cuales una empresa efectúa transacciones de productos o servicios. Los indicadores del capital relacional son entre otros, la participación en el mercado, las tasas de retención y la rentabilidad por cliente (Bautzer, 2010; Stewart, 1998).
El conocimiento sobre los clientes puede transformarse en capital relacional siempre y cuando se responda en forma flexible, rápida y dinámica a las necesidades que éstos tengan. La clave es lograr una relación "ganar-ganar», en la que todas las partes consiguen identificar soluciones satisfactorias para sus problemas, alcanzando los objetivos deseados mediante acuerdos satisfactorios (Stewart, 1998).

\section{Dato, información y conocimiento}

Una tarea previa a la revisión del concepto de conocimiento consiste en distinguir entre dato, información y conocimiento, aunque no exista un consenso sobre los límites entre ellos. Según Davenport y Prusak (1998), los datos son un conjunto discreto de factores objetivos sobre un hecho real. En un contexto empresarial, un dato se refiere a un registro de transacciones $\mathrm{y}$, normalmente, las organizaciones los almacenan en algún tipo de sistema tecnológico. Pero aunque los datos describen únicamente una parte de lo que pasa en la realidad y no proporcionan juicios de valor, son materias primas esenciales para la generación de información, la cual surge cuando se añade a los datos un significado y se organizan para algún propósito. Entonces, la información es un proceso de estructuración del conjunto de datos dotándoles de significado para un sujeto y en un momento concreto. Sin embargo, cuando a esa información se mezclan la experiencia, valores importantes, información contextual y puntos de vista de expertos, facilitando un marco de análisis para la evaluación e incorporación de nuevas experiencias e información, se convierte en conocimiento; así, el conocimiento es más amplio, profundo y rico que los datos o la información.

El conocimiento es un recurso ilimitado ya que es propio de cada individuo (Bueno, 2000), pues se logra enriquecerlo a través de las experiencias profesionales y personales que vive cada persona. Su principal ventaja es que tiene muchas perspectivas, siempre está en constante cambio, evoluciona y hace posible la adaptabilidad de la organización hacia su medio, considerando el tipo de problema, la época y la organización misma.

\section{Gestión del conocimiento}

«Gestión del conocimiento» es un término que en los últimos años ha tenido gran auge, pero ha existido desde la década de los 80's. Para Drucker (1993), es la forma en que las empresas generan, comunican y 
aprovechan sus activos intelectuales, mientras que para Andersen (1999) es la necesidad de acelerar el flujo de la información que tiene valor, desde los individuos de la organización y de vuelta a los individuos, de modo que puedan usarla para crear valor para los clientes. Por su parte, Bueno (2000) la concibe como la función que planifica, coordina y controla los flujos de conocimientos que se producen en la empresa en relación con sus actividades y su entorno, con el fin de crear competencias esenciales. En este sentido, la gestión del conocimiento describe los esfuerzos organizacionales orientados a asegurar que el capital intelectual se encuentre en continuo movimiento, amplificación, venta o compartición, y que además genere resultados organizacionales superiores a los obtenidos por las empresas competidoras.

Una característica esencial del conocimiento, como capital intelectual, es que únicamente genera valor para la empresa cuando se emplea de modo efectivo, por lo que a diferencia del capital financiero, el capital intelectual mantenido en una reserva sin ser utilizado rara vez crea valor económico (De Long y Seeman, 2000). Por esta razón, es importante que la empresa entienda cuál debe ser el ciclo básico de la gestión del conocimiento, con el fin de que desarrolle un proceso dinámico de generación de flujos de conocimiento y de creación de conocimientos nuevos, pero relacionados con su estrategia y actividades. Para Bueno (2000), esta gestión debe saber diseminar el conocimiento por toda la organización e incorporarlo a los productos, servicios, sistemas, procesos y, en suma, convertirlos en determinadas «competencias distintivas». Solo así será posible la creatividad que transforme en innovación y en emprendimiento (Gupta, 2012).

\section{Transferencia del conocimiento}

Se entiende por transferencia del conocimiento el intercambio del mismo, que tiene lugar entre dos unidades dentro de una empresa (transferencia interna) o entre distintas empresas (transferencia externa). En este sentido, Argote e Ingram (2000) enfatizan la experiencia y el aprendizaje, entendiendo por transferencia de conocimiento «el proceso a través del cual una unidad (individuo, grupo, departamento o división) es afectada por la experiencia de otra». Cuando la transferencia del conocimiento traspasa los límites de una organización (transferencia externa) favorece a que las empresas aprendan no solo de su propia experiencia, sino también indirectamente de otras organizaciones (Argote, Beckman y Epple, 1990).

Schulz (2001) apunta una serie de beneficios derivados de los flujos de conocimiento, ya que su transferencia facilita la transmisión del know-how (saber hacer), el cual agiliza la coordinación del trabajo y la explotación de economías de escala. Sin embargo, la utilidad de la creación de conocimiento está limitada por la posibilidad de compartirlo.

\section{Metacognición}

Existen organizaciones en la actualidad que mantienen la falsa percepción de que la acumulación de datos e información, aún sin procesar, les genera conocimiento, y que limitarse a resolver problemas es llevar a cabo procesos exitosos de gestión del conocimiento. Sin embargo, para crear nuevo conocimiento con valor es necesario que cada individuo procese y haga propio el conocimiento que esté adquiriendo, para que pueda ser aplicable en cualquier situación y/o problema que se le presente.

El conocimiento de las distintas operaciones mentales y saber cómo, cuándo y para qué deben usarse, es el objeto de estudio de la metacognición, palabra que significa «más allá del conocimiento». Para Burón (1988), quizá sería mejor llamarlo «conocimiento autorreflexivo», puesto que se refiere al conocimiento de la propia mente adquirida por auto observación. Por lo tanto, la metacognición es el conocimiento que todo individuo tiene de sus operaciones mentales: qué son, cómo se realizan, cuándo hay que usar una u otra, así como qué factores ayudan o interfieren en la operatividad, Flavell (1978).

\section{Gestión del conocimiento y ventaja competitiva}

El uso adecuado del conocimiento es la principal fuente que marca la diferencia en un mercado cada vez más competitivo y global para las organizaciones (Grant, 1991). Por lo tanto, si una organización desea ser competitiva de forma sostenida en el tiempo, deberá identificar, crear, almacenar, transmitir y utilizar de forma eficiente el conocimiento individual y colectivo de sus trabajadores con el fin de resolver problemas, mejorar procesos o servicios y aprovechar nuevas oportunidades de negocio. Entre los beneficios potenciales de la gestión del conocimiento se tiene la mejora de competencias, la eficiencia en procesos y operaciones, la toma de decisiones adecuadas, el 
aprendizaje y la innovación. Todas estas son acciones que se traducen ventajas competitivas (Davenport y Prusak, 2001), por lo que resulta interesante verificar si las empresas u organizaciones consideradas como exitosas en sus respectivos mercados, administran adecuadamente su conocimiento.

\section{Planteamiento del problema}

No se dispone de información acerca de cómo se transforma el conocimiento local e individual en conocimiento organizacional ni de qué manera adquieren, usan y transfieren el conocimiento, y tampoco si realmente cuentan con información suficiente para el diseño de políticas que les hayan permitido ser consideradas como exitosas en sus mercados, ya que no contar con dicha información podría dar lugar a problemas de planeación y falta de políticas de apoyo gubernamental adecuadas que generen un fuerte impacto social a través del uso activo y sistemático del conocimiento. Ante lo expuesto, surgen las siguientes preguntas de investigación: ¿cómo adquieren, generan, usan y transfieren las organizaciones exitosas su conocimiento? ¿Corresponde la gestión del conocimiento a su éxito en su mercado? Para dar respuesta a estas interrogantes, el presente estudio se desarrolló en empresas y organizaciones sociales productivas con sede en Mérida, Yucatán, en el Sureste de México.

\section{Beneficios del estudio}

La información generada abrirá una oportunidad a las instituciones de educación superior, a los centros de investigación científica, a las cámaras empresariales, a los despachos de consultoría y al Gobierno (federal, estatal y municipal) para diseñar e implementar programas de consultoría y capacitación para ayudar a las organizaciones a ser más productivas con base en la gestión del conocimiento, de manera que se mantengan en el mercado. A través de una mejor gestión del conocimiento será posible el fomento de la creatividad, la innovación y el liderazgo efectivo que lleve a las organizaciones a ser flexibles y a sobrevivir a un mundo cada vez más exigente. Además, aunque estos resultados son válidos solo para la población en estudio, la metodología es replicable en otros contextos, ya sean estados o regiones.

\section{Limitaciones}

Esta investigación se llevó a cabo en empresas y organizaciones sociales productivas ubicadas en la ciudad de Mérida, Yucatán, debido a que en ella se desarrolla cerca del $75 \%$ de la actividad empresarial y productiva de la entidad. El trabajo abarca hasta la elaboración de propuestas que permitan a las organizaciones ser gestoras de su propio conocimiento, con base en la información recabada y en lo indicado por los autores citados en este documento. La información de campo se recabó entre abril y mayo de 2011.

\section{MATERIAL Y MÉTODO}

El estudio es de enfoque cualitativo y consta de dos fases. En la primera se identifican las actividades de gestión del conocimiento según los lineamientos expuestos en la revisión de la bibliografía. En la segunda se evalúa, mediante y el uso de escalas, la forma como las empresas llevan al cabo esta gestión.

\section{Tipo y diseño}

En la primera fase. Es de tipo exploratorio y descriptivo, con diseño no experimental transversal (Hernández, Fernández y Baptista, 2010). Como método se recurrió al trabajo de campo y como técnica se utilizó la entrevista.

La segunda fase, también cualitativa, fue descriptiva y con diseño no experimental transversal, recurriendo al trabajo de campo como método y utilizando la entrevista.

\section{Participantes en el estudio}

En la primera fase aceptaron participar los directivos o responsables de empresas de reconocido éxito en la ciudad de Mérida, todas ellas con sucursales y operaciones en todo el Estado de Yucatán. Fueron seleccionadas previa consulta a dos representantes de la Cámara Nacional de la Industria de Transformación, dos de la Cámara Nacional de Comercio, Servicios y Turismo de Mérida, uno de turismo, uno de la cámara de restauranteros y dos del ramo maquilador, así como a tres consultores de negocios, todos ellos considerados como "expertos» en este trabajo. Se buscaba que en las empresas hubiera interés en la gestión del conocimiento, que el directivo fuera mexicano y que tuvieran operación en al menos dos localidades del estado de Yucatán. Las OSP, con operación en el Estado pero con sede en Mérida, fueron seleccionadas según las indicaciones de tres consultores especializados en ellas. Dicha selección fue verificada por el Director del Consejo de Ciencia y Tecnología del Estado de Yucatán (CONCYTEY), luego de lo cual se les invitó formalmente 
a participar. Originalmente fueron 28 organizaciones, pero solamente estas 24 accedieron.

Aunque no se consideró originalmente el tamaño, resultó que todas las empresas califican como grandes, pero las OSP resultaron ser medianas según los criterios del Diario Oficial de la Federación (2009). Salvo una maquiladora de exportación y una cooperativa pesquera especializada en pulpo, todas las demás tienen su sede en Mérida, quedando la distribución como sigue:

\section{Manufactureras}

M1. Intercambiadora de calor de aluminio para la industria automotriz.

M2. Embotelladora de bebidas gaseosas.

M3. Fábrica de galletas y productos de harina.

M4. Constructora especializada en vivienda.

M5. Maquiladora textil de exportación.

M6. Fabricante de prótesis dentales con calidad de exportación.

\section{Comerciales}

C1. Venta de productos de harina para consumo humano.

C2. Venta de artículos al por menor. Autoservicio, artículos varios.

C3. Venta de artículos al por menor. Supermercado.

C4. Venta de artículos al por mayor.

C6. Venta de ropa.

C7. Venta de materiales para construcción.

\section{Servicios}

S1. Servicios de mercadotecnia.

S2. Servicio de medios de comunicación masiva.

S3. Hotel de cadena internacional.

S4. Hotel de cadena nacional.

S5. Restaurante de comida típica.

S6. Restaurante de comida internacional.

S7. Aseguradora.

S8. Agencia de viajes.

\section{Organizaciones sociales productivas}

OSP 1. Cooperativa apícola.

OSP 2. Cooperativa apícola.

OSP 3. Cooperativa pesquera especializada en pulpo.

OSP 4. Caja popular.

En la segunda fase se pidió a cada uno de los expertos, en una nueva visita, que evaluara las empresas de su ámbito de competencia según las escalas.

\section{Instrumentos y procedimientos}

En la primera fase se utilizó un instrumento, el cual fue una guía de entrevista semiestructurada. Las preguntas (ítems) son de respuesta abierta y se incluyen preguntas generales sobre la adquisición, uso y transferencia del conocimiento, así como dos reactivos de respuesta cerrada. De la pregunta 5 a la 12 se tomaron del Manual de Bogotá presentado por Jaramillo, Lugones y Salazar (2001), para efecto de interpretación de los datos obtenidos en dichas preguntas. La 5 de este instrumento es la 38 de dicho manual, la 6 es la 44 , la 7 es la 82 , la 8 es la 83 , la 10 es la 84 , la 11 es la 85 y la 12 es la 86 , respectivamente. La clasificación de los resultados siguió el concepto de gestión del conocimiento de Drucker (1993) primordialmente, poniendo atención especial al modelo de Nonaka y Takeuchi (1995). Se incluyeron preguntas que detectaran procesos de transferencia a nivel empresa y personal, así como el fomento de la metacognición (Flavell, 1978). Al final se incluyó una sección con preguntas abiertas generales.

El instrumento fue revisado por tres expertos en gestión del conocimiento para verificar la validez de contenido (Hernández et al., 2010). Se eligió la guía de entrevista debido a la posibilidad de obtener información más abundante sobre los conceptos referidos. Dichas entrevistas fueron administradas por el investigador responsable de este proyecto y dos tesistas de maestría dedicadas a este trabajo, con la capacitación previa correspondiente. En todo momento se contó con el apoyo del CONCYTEY para los permisos y cartas de apoyo pertinentes. Cada entrevista duró entre 45 y 60 minutos.

Las respuestas abiertas fueron registradas clasificándolas por frecuencia de mención (frases testimonio) representándolas con ideas clave (ÁlvarezGayou, 2003) con los respectivos porcentajes de frecuencia de respuestas, tanto en lo general como por giro. Para cálculos numéricos y la elaboración de los cuadros se emplearon las utilerías de Excel.

Con base en los resultados se construyeron escalas de valoración tipo rúbricas para que sirvieron en la segunda fase para que el autor de este trabajo y las tesistas evaluaran en conjunto a cada una de las empresas participantes en lo correspondiente a adquisición, uso y transferencia de conocimiento (ver tabla I). 


\section{RESULTADOS}

\section{Adquisición del conocimiento}

Las actividades que realizan las empresas y OSP para adquirir conocimiento son principalmente la capacitación externa $(20.7 \%)$, seguida por la capacitación impartida por proveedores, la capacitación interna, las investigaciones de mercado y los procesos administrativos. Las menos recurridas, ambas con $6.9 \%$ de las menciones, se refieren a métodos y procedimientos de operación y a investigaciones de mercado, indicando que se consume el conocimiento que traen otros, siendo poco o nulo el que se genera en las empresas. Sin embargo, al seccionar por giro, las manufactureras recurren a capacitación de proveedores principalmente, mientras que las de servicio y las comerciales recurren a consultores externos. Para las OSP, obtienen su conocimiento, especialmente el de tipo científico, a partir de instituciones de gobierno y centros de investigación (20\%).

El $23 \%$ de las empresas participantes no adquiere conocimiento proveniente de instituciones públicas o de centros de investigación científica (ClCs), y las que lo hacen recurren más frecuentemente a la Universidad Autónoma de Yucatán (UADY) o al Centro de Investigación Científica de Yucatán (CICY), lo cual

TABLA I

EVALUACIÓN DE LA GESTIÓN DEL CONOCIMIENTO POR PARTE DE LAS EMPRESAS

\begin{tabular}{|c|c|c|c|c|c|}
\hline \multirow[b]{2}{*}{ Manufactureras } & \multicolumn{3}{|c|}{ Conocimiento } & \multirow[b]{2}{*}{ Promedio } & \multirow[b]{2}{*}{ Resultado } \\
\hline & Adquisición & Uso & Transferencia & & \\
\hline M1. Intercambiadora de calor de aluminio para la industria automotriz. & 3 & 3 & 2 & 2.7 & Muy bien \\
\hline M2. Embotelladora de bebidas gaseosas. & 3 & 4 & 4 & 3.7 & Excelente \\
\hline M3. Fábrica de galletas y productos de harina. & 4 & 4 & 4 & 4.0 & Excelente \\
\hline M4. Constructora especializada en vivienda. & 3 & 3 & 3 & 3.0 & Muy bien \\
\hline M5. Maquiladora textil de exportación. & 3 & 4 & 2 & 3.0 & Muy bien \\
\hline M6. Fabricante de prótesis dentales con calidad de exportación. & 4 & 4 & 3 & 3.7 & Excelente \\
\hline \multicolumn{6}{|l|}{ Comercial } \\
\hline C1. Venta de productos de harina para consumo humano. & 3 & 4 & 4 & 3.7 & Excelente \\
\hline C2. Venta de artículos al pormenor. Autoservicio, artículos varios. & 2 & 4 & 2 & 2.7 & Muy bien \\
\hline C3. Venta de artículos al por menor. Supermercado. & 2 & 3 & 1 & 2.0 & Regular \\
\hline C4. Venta de artículos al por mayor. & 3 & 3 & 1 & 2.3 & Regular \\
\hline C5. Venta de ropa. & 3 & 3 & 1 & 2.3 & Regular \\
\hline C6. Venta de materiales para construcción. & 2 & 2 & 1 & 1.7 & Regular \\
\hline \multicolumn{6}{|l|}{ Servicios } \\
\hline S1. Servicios de mercadotecnia. & 3 & 3 & 2 & 2.7 & Muy bien \\
\hline S2. Servicio de medios de comunicación masiva. & 3 & 3 & 3 & 3.0 & Muy bien \\
\hline S3. Hotel de cadena internacional. & 4 & 4 & 3 & 3.7 & Excelente \\
\hline S4. Hotel de cadena nacional. & 3 & 4 & 2 & 3.0 & Muy bien \\
\hline S5. Restaurante de comida típica. & 2 & 3 & 2 & 2.3 & Regular \\
\hline S6. Restaurante de comida internacional. & 3 & 4 & 2 & 3.0 & Muy bien \\
\hline S7. Aseguradora. & 4 & 4 & 3 & 3.7 & Excelente \\
\hline S8. Agencia de viajes. & 4 & 3 & 2 & 3.0 & Muy bien \\
\hline \multicolumn{6}{|l|}{ Organizaciones sociales productivas } \\
\hline OSP1. Cooperativa apícola. & 2 & 3 & 2 & 2.3 & Regular \\
\hline OSP2. Cooperativa apícola. & 2 & 2 & 1 & 1.7 & Regular \\
\hline OSP3. Cooperativa pesquera especializada en pulpo. & 2 & 3 & 1 & 2.0 & Regular \\
\hline OSP4. Caja popular. & 1 & 2 & 1 & 1.3 & Deficiente \\
\hline
\end{tabular}

Fuente: Datos de la investigación. Interpretación: De 0 a 0.5= muy deficiente / 0.6 a $1.5=$ deficiente / 1.6 a $2.5=$ regular $/ 2.6$ a 3.5 = muy bien / 3.6 a $4=$ excelente 
se repite para las manufactureras y comerciales. En el caso de las últimas, la vinculación con el CICY se relaciona con desarrollo de productos. Llama la atención que ninguna de las OSP hubiera indicado que se vincula con IES o con CICs.

De manera general, las empresas no recurren a instancias gubernamentales, o si lo hacen, con mayor frecuencia participan en talleres de gobierno organizados por instancias estatales primordialmente, así como federales. El conocimiento que adquieren en estas actividades se relaciona con la operación del negocio, y en el caso de las OSP, obtienen también información técnica para el desarrollo de sus actividades. El Instituto Nacional para la Innovación, la Calidad y la Competitividad (INICC) se encarga de ofrecer cursos de calidad y mejora continua.

Las empresas manufactureras y la mitad de las de servicio obtienen conocimiento a través de cámaras y despachos dedicados a calidad, aunque un número significativo de las comerciales no recurren a estas instancias. En el caso de las OSP, sus directivos manifestaron que obtienen conocimiento científico y general por medio de otras personas que realizan la actividad, es decir, aprovechan la experiencia de otros, lo que reafirma la importancia del comportamiento tácito y hacerlo explícito (Nonaka y Takeuchi, 1995).

\section{Uso del conocimiento}

Los beneficios del uso del conocimiento son mayor tecnología, ayuda para prever y adaptase al medio, mejorar el servicio al cliente, estandarizar procesos, desarrollar nuevos conocimientos y nuevos productos. Enel caso de las OSP, las experiencias más positivas han sido el desarrollo de nuevos procesos, conocimientos y herramientas para adaptarse al medio y sobrevivir ante la incertidumbre. Todos los entrevistados reconocieron que usar el conocimiento en la organización ayuda a la productividad, lo que concuerda con lo establecido por Davenport y Prusak (2001) en cuanto a los beneficios sobre la gestión del conocimiento.

Los resultados arrojan que el uso principal del conocimiento en las empresas y las OSP es para capacitación, seguida de investigación y desarrollo, y con menor frecuencia para cambios organizacionales. Las únicas empresas que se vinculan con instituciones de educación superior (IES), CICs y cámaras para el uso del conocimiento son las manufactureras. La principal vinculación de todos los giros es con las IES públicas (20.6\%), y con el Consejo Nacional de Ciencia y Tecnología (CONACYT) es el $11.8 \%$, a través de programas de Fondos Mixtos o de apoyo a la innovación. En el caso de las OSP, la vinculación más frecuente es con instancias gubernamentales de apoyo a la producción (50\%).

El uso del conocimiento para la publicación en revistas científicas y de divulgación, ya sea por parte de las empresas o de investigadores que se refieran a ellas, es escaso o nulo. La adquisición y generación de patentes y franquicias también es escasa o nula, salvo para el $26.7 \%$ de las empresas de manufacturas.

\section{Transferencia del conocimiento}

Para los participantes, la transferencia de conocimiento se efectúa principalmente a través de juntas de trabajo (44.4\%) y capacitación impartida por los miembros más experimentados de la empresa (37\%). Sin embargo, en las manufactureras se hizo evidente la transmisión de conocimiento tácito para hacerlo explícito a través de sesiones de lluvia de ideas, aunque con un bajo porcentaje de menciones (7.4\%). Faltan mecanismos de transferencia de conocimiento hacia el exterior, ya que el $52.9 \%$ de los participantes manifestaron que sus empresas no tienen transferencia externa. Se quedan con su propio conocimiento y con el que adquieren, denotándose cierto celo por compartirlo.

En cuanto a transferencia de conocimiento a través de franquicias, solo un pequeño porcentaje las ha otorgado $(6.7 \%)$, y esto corresponde a una de las empresas manufactureras participantes en el estudio. Su experiencia ha sido exitosa y le ha ayudado a expandir su negocio más allá del estado de Yucatán, inclusive al extranjero.

Las únicas empresas en el estudio sin procesos certificados fueron las comerciales. En las otras, la mayor parte de las que sí tienen algún certificado, éste se trata de ISO. La mitad de las empresas de servicio son restaurantes o tienen un restaurante, y todas ellas cuentan con el Distintivo $\mathrm{H}$, certificado de calidad en elaboración de alimentos. En cuanto a productos certificados, las únicas son las mieleras y un fabricante de bebidas gaseosas con su etiqueta biodegradable, constituyendo el $86.7 \%$ de las respuestas Ninguna de las demás participantes cuenta con productos propios certificados.Tampoco se favorece la metacognición 
(Flavell, 1978) y, por ende, si el conocimiento no se hace propio, no se puede transferir.

\section{Comentarios generales sobre gestión del conocimiento}

La relación percibida por los participantes entre gestión del conocimiento, tanto el general como el científico, y los resultados de la organización, son mayor productividad (62.5\%), más utilidades $(25.0 \%)$, menor rotación del personal al permitir la creatividad y participación en la innovación (8.3\%), llegando a operar con más calidad $(4.3 \%)$. Todos manifestaron que la relación más fuerte es entre la gestión del conocimiento y la productividad, generando mayores utilidades. La gestión del conocimiento en general, y del científico en particular, ayudan a las empresas a ser más productivas y rentables, lo que refuerza lo expresado por Drucker (1993) y Agudelo (2011). Otros beneficios son que la gestión del conocimiento ayuda al desarrollo general $(35.7 \%)$, permite la entrada y asimilación de nueva tecnología $(28.6 \%)$, ayuda a estar a la vanguardia (21.4\%) y, por supuesto, a entender los procesos y su mejora continua (14.3\%).

La mayoría de los participantes $(66.7 \%)$ reconoce que hay relación entre la gestión del conocimiento y la tecnología ya que una lleva a la otra. Sin embargo, Ilama la atención el que la tercera parte de las menciones (33.3\%) sostuvieran que no hay relación entre estos dos conceptos, lo cual puede deberse principalmente a que no tienen claro lo que es el conocimiento ni la gestión de éste.

Existe desconocimiento general con respecto a los programas y beneficios que tienen los CICs (61.1\%), lo cual es resultado de la falta de vinculación efectiva entre los sectores académico y productivo. Entre los programas conocidos para fomentar la innovación están los del CICY (25\%), CONACYT (20\%), SAGARPA(25\%) y CONCYTEY (5\%). Sin embargo, la mayoría de los participantes no conoce los apoyos o programas con que cuenta el Gobierno para apoyo a la innovación, lo que denota la falta de vinculación entre los sectores gubernamental y productivo.

\section{Evaluación de la gestión del conocimiento de las empresas y OSP participantes}

Con base en los resultados y la revisión de la literatura, el autor de este trabajo construyó estas escalas (rúbricas) para evaluar, junto con las tesistas, lo que cada empresa u OSC participante realiza (ver tabla II, III y IV), según lo que una organización debe hacer en cuanto a gestión del conocimiento (Drucker, 1993; Bautzer, 2010; Agudelo, 2011).

Todas las organizaciones tienen alguna forma de administrar su conocimiento, ya sea adecuadamente $o$ no. Las que lo adquieren, usan y transfieren según lo

TABLA II

ESCALA (RÚBRICA) PARA VALORAR LA ADQUISICIÓN DE CONOCIMIENTO

\begin{tabular}{|c|c|c|c|c|}
\hline Excelente & Muy bien & Regular & Deficiente & Muy deficiente \\
\hline $\begin{array}{l}\text { Realiza la empresa } \\
\text { actividades para } \\
\text { adquirir } \\
\text { conocimiento, se } \\
\text { vincula con IES y } \\
\text { CICs para adquirir } \\
\text { conocimiento. } \\
\text { Se vincula con } \\
\text { cámaras y } \\
\text { despachos, } \\
\text { además de que } \\
\text { genera } \\
\text { conocimiento } \\
\text { propio. }\end{array}$ & $\begin{array}{l}\text { Realiza } \\
\text { actividades } \\
\text { frecuentes para } \\
\text { adquirir } \\
\text { conocimiento. } \\
\text { Tiene alguna } \\
\text { vinculación con } \\
\text { otros } \\
\text { organismos, } \\
\text { pero no genera } \\
\text { su propio } \\
\text { conocimiento. }\end{array}$ & $\begin{array}{l}\text { Realiza } \\
\text { algunas } \\
\text { actividades } \\
\text { para adquirir } \\
\text { conocimiento. } \\
\text { Su vinculación } \\
\text { es escasa y no } \\
\text { genera su } \\
\text { propio } \\
\text { conocimiento. }\end{array}$ & $\begin{array}{l}\text { El conocimiento } \\
\text { viene } \\
\text { prácticamente } \\
\text { de proveedores } \\
\text { y capacitadores. } \\
\text { Escasa o nula } \\
\text { vinculación. }\end{array}$ & $\begin{array}{l}\text { El conocimiento } \\
\text { viene de } \\
\text { externos y no } \\
\text { hay vinculación } \\
\text { con otros } \\
\text { organismos. }\end{array}$ \\
\hline 4 & 3 & 2 & 1 & 0 \\
\hline
\end{tabular}


TABLA III

ESCALA (RÚBRICA) PARA VALORAR EL USO DEL CONOCIMIENTO

\begin{tabular}{|c|c|c|c|c|}
\hline Excelente & Muy bien & Regular & Deficiente & Muy deficiente \\
\hline $\begin{array}{l}\text { La empresa usa } \\
\text { adecuadamente su } \\
\text { conocimiento, se } \\
\text { vincula con IES y } \\
\text { CICs para dicho uso. } \\
\text { Logra patentes o } \\
\text { licencias. }\end{array}$ & $\begin{array}{l}\text { Usa su } \\
\text { conocimiento } \\
\text { para incrementar } \\
\text { su } \\
\text { competitividad. } \\
\text { Tiene alguna } \\
\text { vinculación con } \\
\text { otros } \\
\text { organismos, y no } \\
\text { genera licencias } \\
\text { ni patentes. }\end{array}$ & $\begin{array}{l}\text { Usa su } \\
\text { conocimiento } \\
\text { para mejorar los } \\
\text { productos o } \\
\text { servicios que ya } \\
\text { realiza, así } \\
\text { como algunos } \\
\text { repuntes para } \\
\text { mejoramiento } \\
\text { de su } \\
\text { operación. } \\
\text { No genera } \\
\text { licencias ni } \\
\text { patentes. }\end{array}$ & $\begin{array}{l}\text { El uso de su } \\
\text { conocimiento es } \\
\text { para la } \\
\text { subsistencia } \\
\text { diaria, es decir, } \\
\text { según lo que pida } \\
\text { la situación. } \\
\text { Poco o nulo } \\
\text { avance } \\
\text { tecnológico } \\
\text { propio y escasa o } \\
\text { nula vinculación. }\end{array}$ & $\begin{array}{l}\text { No se aprovecha } \\
\text { el conocimiento } \\
\text { para operar } \\
\text { adecuadamente. } \\
\text { Se deja en } \\
\text { experiencia } \\
\text { empírica y de } \\
\text { subsistencia. } \\
\text { Nula } \\
\text { vinculación. }\end{array}$ \\
\hline 4 & 3 & 2 & 1 & 0 \\
\hline
\end{tabular}

TABLA IV

RÚBRICA PARA VALORAR LA TRANSFERENCIA DE CONOCIMIENTO

\begin{tabular}{|c|c|c|c|c|}
\hline Excelente & Muy bien & Regular & Deficiente & Muy deficiente \\
\hline $\begin{array}{l}\text { La empresa } \\
\text { transfiere } \\
\text { adecuadamente su } \\
\text { conocimiento, se } \\
\text { vincula con IES y } \\
\text { CICs para dicha } \\
\text { transferencia }\end{array}$ & $\begin{array}{l}\text { Transfiere su } \\
\text { conocimiento de } \\
\text { manera } \\
\text { suficiente, tanto } \\
\text { interna como } \\
\text { externamente. } \\
\text { Tiene alguna } \\
\text { vinculación con } \\
\text { otros organismos }\end{array}$ & $\begin{array}{l}\text { Transfiere su } \\
\text { conocimiento } \\
\text { de manera } \\
\text { insuficiente. } \\
\text { Mayor énfasis } \\
\text { en transferencia } \\
\text { interna que } \\
\text { externa. Poca } \\
\text { vinculación con } \\
\text { otros } \\
\text { organismos. }\end{array}$ & $\begin{array}{l}\text { Transfiere su } \\
\text { conocimiento de } \\
\text { manera } \\
\text { insuficiente. } \\
\text { Énfasis } \\
\text { únicamente en } \\
\text { transferencia la } \\
\text { interna, si es que } \\
\text { existe. } \\
\text { Escasa o nula } \\
\text { vinculación con } \\
\text { otros } \\
\text { organismos. }\end{array}$ & $\begin{array}{l}\text { No hay } \\
\text { transferencia ni } \\
\text { interna ni } \\
\text { externa. } \\
\text { No hay } \\
\text { vinculación con } \\
\text { otros } \\
\text { organismos. }\end{array}$ \\
\hline 4 & 3 & 2 & 1 & 0 \\
\hline
\end{tabular}


requiere su medio, son las que sobreviven, crecen y se desarrollan. Las que no aprenden de su experiencia, no conocen a sus clientes o usuarios y no transfieren el conocimiento son las que perecen. Desde este punto de vista, una adecuada gestión del conocimiento es aquella que permite a la organización alcanzar sus metas, y en la medida en que lo hagan eficientemente, se adaptarán a los cambios y permanecerán en el mercado.

Los participantes reconocen que la adecuada gestión del conocimiento ayuda al desarrollo organizacional, por lo que la gestión del conocimiento es una inversión si dicho conocimiento se orienta a mejoras en la productividad. De hecho, a nivel empresa y principalmente en las manufactureras y las de servicios, el desempeño exitoso que presentan corresponde a una gestión del conocimiento muy buena o excelente. Se enfrentan a mercados competitivos, clientes con expectativas exigentes y cambiantes, estando constantemente sujetas a procesos de acreditación, por lo que tienen que adquirir, usar y transferir conocimiento, participar en ferias, vincularse con IES e innovar constantemente. Su gestión del conocimiento está orientada a la adquisición, uso y transferencia de manera adecuada y constante, lo que es propicio para la innovación y el emprendimiento, tal como indica Gupta (2012). Por otra parte, en las comerciales hay que mejorar los mecanismos de transferencia, cuyas bajas calificaciones pueden deberse al celo por compartir lo que hacen y a la gran competencia que enfrentan en mercados regidos principalmente por el precio. Tal parece que adquieren y usan el conocimiento, pero se quedan con él y no lo comparten ni aprenden de otras empresas en sus mercados (transferencia externa) ni lo transmiten internamente. Asi mismo, se orienta a adquirir y operar, pero poco a transferir y aprender de otros y del conocimiento tácito de los empleados, lo que resulta en operación eficiente, pero limitada innovación.

En cuanto a las OSP, el desempeño exitoso solo se vinculó con una gestión del conocimiento regular o deficiente. Adquieren conocimiento de manera limitada, al parecer por procedimientos estandarizados por los dirigentes de las cooperativas, pero enfrentan poca competencia. Da la impresión de que consideran que el conocimiento que tienen es suficiente, por lo que operan en una zona de estabilidad o confort que les brinda seguridad en su mercado. Ese conocimiento lo usan de manera «regular» a «muy buena», pero no se ven esfuerzos por transferir y compartir el conocimiento. Su gestión del conocimiento está orientada a adquirir lo necesario y a usarlo adecuadamente, con poca o limitada innovación. Y en cuanto a la caja popular, su éxito al parecer se debe más a que responde a una necesidad del público que a sus esquemas de gestión conocimiento, por lo que aunque sea deficiente en general, es suficiente para permanecer. Su gestión del conocimiento está orientada a operar, pero con limitada orientación a la innovación, prevaleciendo la importancia hacia el capital estructural (Bautzer, 2010) y relacional, sin incrementos significativos del capital intelectual.

En la medida en que las organizaciones administren eficaz y eficientemente su conocimiento, se adaptarán al medio y serán exitosas en cuanto a que cumplirán sus metas, confirmando que una adecuada gestión del conocimiento lleva a la permanencia en el mercado. No se puede hablar de creatividad, innovación y emprendimiento sin una gestión del conocimiento que permita generar, adquirir, usar y transferir valor al mercado y a la sociedad. Si bien es cierto, que el conocimiento es el único recurso ilimitado en una organización, la fuerza que lo mueve es la voluntad. Con visión, capacidad de gestión y voluntad, el conocimiento se convertirá en valor y ventajas competitivas, con lo que será posible lograr las empresas y organizaciones productivas que México necesita. 


\section{REFERENCIAS}

Agudelo, M. A. (2011). Rolestratégico de la universidad en la construcción de conocimiento para el entorno global. En A.M. Sánchez y M. C. Corrales (Eds.). Administración de conocimiento y desarrollo basado en conocimiento. Redes e innovación. México: Cengage.

Álvarez-Gayou, J. (2003). Cómo hacer investigación cualitativa. Fundamentos y metodología. México: Paidós Mexicana. Colección Paidós Educador

Andersen, A. (1995). Modelo de la American Productivity \& Quality Center. Recuperado el 6 de junio de 2011 de http://www.exa.unicem.edu.ar

Argote, L.; Beckman, S.L. y Epple, D. (1990, febrero). The persistence and transfer of learning in industrial settings. Management Science, 36 (2), 140-155.

Argote, L. e Ingram, I. (2000, mayo). Knowledge transfer: a basis for competitive advantage in firms. Organizational Behaviour and Human Decision Processes, 82 (1). 150-169.

Argyris, C. (1992). On organizational learning (2ª Ed). Malden (EUA): Blackwell.

Barroso, F. (2011). Gestión del conocimiento. Un estudio exploratorio en empresas de la ciudad de Mérida, Yucatán. Memorias del XXIV Congreso de la Sociedad Latinoamericana de Estrategia (SLADE). Mérida Yucatán, mayo 18 al 21. Universidad Anáhuac Mayab.

Bautzer, D. (2010). Capital Intelectual. Revista Innovación Educativa, 10 (51), 15-19.

Bueno, E. (2000). La gestión del conocimiento en la nueva economía en gestión del conocimiento $y$ capital intelectual. Experiencias en España. Madrid, España: Instituto Universitario Euroforum Escorial.

Burón, O. (1988). Enseñar a aprender: Introducción a la Metacognición (4 ${ }^{a}$. ed.). España: Ediciones Mensajero.

Castells, M. (1997). The power of identity. Oxford: Blackwell.

Davenport, T.H. y Prusak, L. (1998). Working knowledge. How organizations manage what they know. E.U.A.: Harvard Business School Press.

De Long D. y Seeman, P. (2000). Confronting conceptual confusion and conflict in knowledge management. Organizational Dynamics, 29, 33-44.

Diario Oficial de la Federación (2009, 30 de junio). Disponible en http://www.ccexpansion. com/emprendedores/2009/06/30/el-gobiernoreclasifica-las.pymes

Drucker, P. F. (1993) Post-Capitalist Society. Nueva York, (E.U.A.): Harper Collins Publishers.

Flavell, J.H. (1978). Metacognitive development. En J.M. Scandura y C.J. Brainerd (eds.). Structural process models of complex human behavior. Holanda: Sifthoff y Noordhoff.

Garvin, D. (2000) Crear una organización que aprende. Deusto, España: Harvard Business School Edic./ Deusto.

Grant, R.M. (1991, primavera). The Resource-Based Theory of competitive advantage: Implications for strategy formulation. California Management Review, 114-135.

Gupta, P. (2012). The Innovation Solution. Making Innovation More Pervasive, Predictable and Profitable. E.U. A.: Accelper Consulting.

Hernández, R.; Fernández, C. y Baptista, L (2010). Metodología de la investigación ( $5^{\mathrm{a}}$. ed.). México: Mc. Graw Hill.

Jaramillo, H.; Lugones, G. y Salazar, M. (2001). Manual de Bogotá para la normalización de indicadores de innovación tecnológica en America Latina y el Caribe. Colombia: Red Iberoamericana de Indicadores de Ciencia y Tecnología (RICYT) / Organización de Estados Americanos (OEA). Colciencias/ OCYT.

Nonaka, I. y Takeuchi, H. (1995). La organización creadora de conocimiento. Nueva York, E.U.A.: Oxford University Press.

Stewart, T. A. (1998). La nueva riqueza de las organizaciones: el capital Intelectual. Argentina: Granica

Schulz, M. (2001). The uncertain relevance of newness: organizational learning and knowledge flows. Academy of Management Journal, 44(4), 660-681. 